\title{
Fair Routing in Delay Tolerant Networks
}

\author{
Josep M. Pujol \\ Telefonica Research, Barcelona \\ e-mail: jmp@tid.es
}

\author{
Alberto Lopez Toledo \\ Telefonica Research, Barcelona \\ e-mail: alopezt@tid.es
}

\author{
Pablo Rodriguez \\ Telefonica Research, Barcelona \\ e-mail: pablorr@tid.es
}

\begin{abstract}
The typical state-of-the-art routing algorithms for delay tolerant networks are based on best next hop hill-climbing heuristics in order to achieve throughput and efficiency. The combination of these heuristics and the social network structure leads the routing to direct most of the traffic through a small subset of good users. For instance, in the SimBet algorithm, the top $10 \%$ of users carry out $54 \%$ of all the forwards and $85 \%$ of all the handovers. This unfair load distribution is not sustainable as it can quickly deplete constraint resources in heavily utilized mobile devices (e.g. storage, battery, budget, etc.). Moreover, because a small number of users carry a significant amount of the traffic, the system is not robust to random failures and attacks.

To overcome these inefficiencies, this paper introduces FairRoute, a routing algorithm for delay tolerant networks inspired by the social processes of perceived interaction strength, where messages are preferably forwarded to users that have a stronger social relation with the target of the message; and assortativity, that limits the exchange of messages to those users with similar "social status". We compare the performance of FairRoute to the state-of-the-art algorithms by extensive simulations on the MIT reality mining dataset. The results show that our algorithm outperforms existing algorithms in the de facto benchmark of throughput vs. forwards. Furthermore, it distributes better the load; the top $10 \%$ carry out $26 \%$ of the forwards and $28 \%$ of the handovers without any loss in performance.
\end{abstract}

\section{INTRODUCTION}

Thanks to the increasing number of mobile devices with wireless capabilities [1], the possibility of communication without network infrastructure is becoming a reality. For example, pocket switched networks rely exclusively on users exchanging messages among themselves upon proximity encounters. Provided that a significant amount of traffic is composed of delay tolerant messages, this paradigm, known also as delay tolerant networks (DTN) [2], could contribute to significantly reduce infrastructure costs and increasing bandwith by orders of magnitude [3].

Because of their potential benefits, there has been a significant body of work in DTNs, with emphasis on routing algorithms. Since the seminal Epidemic routing algorithm [4] was introduced, there has been a succession of algorithms aimed at maximizing throughput - the number of messages arriving to the destination - while minimizing the traffic overhead caused by the number of forwards. While Epidemic routing achieves the optimal throughput, it is very inefficient: multiple copies of a message are forwarded until the network is flooded with redundant messages. Although there have been proposals to reduce the network overhead of flooding
[5], current algorithms do not duplicate messages in the network. This single-copy strategy requires routing algorithms to implement a next-best-hop heuristic which forwards the messages to those nodes with a highest probability to deliver the message to its destination.

Some algorithms operate under the assumption that the network of contacts is known a priori [6]. However, most recent algorithms rely only on mobility [7], [8], [9], [10], [11]. The availability of good quality datasets with contact traces gathered from real human mobility patterns [12], [13], has spurred a breed of sophisticated routing algorithms that exploit the topological properties of social networks [14], [15] to compute the next-best-hop heuristics.

For instance, SimBet [16] relies on social distance based on transitivity [17] and betweenness centrality [18]. Also, Bubble [19] takes advantage of the empirically observed community structure in social networks [20] to decide which heuristic is more likely to be effective depending on the social group of the destination.

\section{MOTIVATION: FAIRNESS WHO?}

Routing in DTNs requires the answer to two questions [21]: 1) how the best next hop is calculated; and 2) how the traffic load is distributed among users. Most of the existing work has focused on maximizing throughput and minimizing number of forwards in order to obtain efficient algorithms with high delivery ratios, but with the exception of [22], [23], there has been no attention to scalability and reliability. However, the intrinsic nature of DTNs, based on both human interaction and mobile phones, makes those networks prone to failures.

Contact traces used by DTN routing algorithms are correlated to social networks [24], [14], and social networks are a particular case of complex networks [25], [26]. One of the characteristics of such networks is that they exhibit a fat-tail connectivity distribution, where few nodes have many connections whereas the majority have very few. Since messages are forwarded via contacts, it is inevitable that the most connected nodes carry the majority of the traffic, hence producing an unfair load distribution. This is true even if traffic is routed at random.

Unfortunately for DTNs, fairness can only worsen if heuristics are applied to the forwarding decisions as we will show next. Let us assume a random forward algorithm. Then the message forwarding process is a random walk over a network with the normalized adjacency matrix $A$. The probability of 
going from node $i$ to node $j$ is $A_{i j}$, which is the inverse of $i$ 's connectivity degree under the random routing assumption. On the other hand, $j$ 's probability of receiving a message is $\sum_{i} A_{i j}$. It trivial to see that nodes with higher connectivity have a higher probability of receiving the message. The probability of finding a message in node $j$, which is equivalent to its traffic load, can be calculated as the first eigenvector of $P^{T}$, where $P_{i, j}=A_{i, j}\left(\sum_{j} A_{i, j}\right)^{-1}$ is the stochastic matrix corresponding to $A$. The eigen vector for adjacency matrices of heterogeneous connectivity distributions will be skewed towards the most connected nodes. Thus, the natural unbalance of the load distribution in complex networks appears. Furthermore, if the forward is not random, but informed by an heuristic that biases towards connectivity, the probability of the highly connected nodes to receive messages is inevitablely increased, and therefore, the load distribution to become even more unbalanced.

In systems that display a similar complex network connectivity such as the Internet, road networks or airline traffic; the unbalance problem is solved by upgrading the resources of the bottleneck nodes, e.g., by installing faster switches, constructing more 8-lane highways or expanding the airports with more terminals. However, this solution cannot be implemented system-wide in DTNs because every single node belongs to a different administrative domain (i.e., individual users). In addition, the mobile phone market is fairly homogeneous in terms of resources consumption, such as battery-life. Therefore, unlike other systems, the natural unbalance due to the network structure cannot be compensated by assuming that all the bottleneck nodes will be upgraded.

Under these circumstances failures can occur by a variety of reasons: 1) nodes do not have enough resources to manage the load and fail [27], 2) important nodes are logic targets of attacks [28]; and 3) the mismatch between the incurred cost (total messages to forward) and the utility (own messages to forward) of the bottleneck nodes is a deterrent for their participation in the network, and these nodes are, ironically, the most needed [29]. These problems illustrate the need of a fair distribution of the traffic load in the routing algorithms of DTNs. As an example, fairness and load balance are recurrent topics in other areas such as wireless sensor networks or Internet [30], [31], [32].

The rest of the paper is organized as follows: in Section III we present FairRoute, our DTN routing algorithm. In Section IV we discuss the empirical dataset used in the simulation as well as the experimental design. Sections V - VII summarize and discuss the results of the experiment in terms of efficiency and throughput (Section V), fairness (Section VI) and goodness of the assortative load control (Section VII). Finally Section VIII presents the conclusions and future work.

\section{FAIRRoute Algorithm}

In DTNs messages are forwarded from node to node upon proximity contacts. Because the trace of contacts is a social network, we turn into social science to design an effective routing algorithm that overcomes the unfairness problem of existing algorithms. In particular FairRoute relies in perceived interaction strength and assortativity to guide the forward decision upon contact.

\section{A. Interaction Strength at Different Time Scales}

The perceived interaction strength, a concept developed from social influence [33] [34], represents the subjective assessment of the strength of a social tie between two individuals. The interaction strength can be used as an indicator of the likelihood of a contact to be sustained over time. FairRoute uses two different estimators of interaction strength that operate at different time scales: $\sigma_{i j}$ that indicates the interaction strength between $i$ and $j$ in the short term; and $\lambda_{i j}$ that indicates the interaction strength in a longer time scale. The strength of the $i j$ interaction increases upon contact but decreases over time at an exponential rate $r_{\sigma}$ and $r_{\lambda}$ for the short term and long term interaction strength respectively. For such reason it is required that $r_{\lambda} \ll r_{\sigma}$.

When a contact between $i$ and $j$ takes place, the nodes update their perceived interaction strengths as follows:

$$
\begin{aligned}
\sigma_{i k} & =\sigma_{i k} e^{-r_{\sigma}\left(t-t_{i}\right)} \quad \forall_{k \in N_{i}}, \\
\lambda_{i k} & =\lambda_{i k} e^{-r_{\lambda}\left(t-t_{i}\right)} \quad \forall_{k \in N_{i}}, \\
\left(\sigma_{i j}, \lambda_{i j}\right) & =\left(\sigma_{i j}, \lambda_{i j}\right)+(1,1),
\end{aligned}
$$

where $N_{i}$ is the list of contacts of node $i, t_{i}$ is the time of node $i$ 's last contact (with any other node), and $t$ is the current time. Upon contact, node $i$ updates the exponential decrease of the perceived strength with all the nodes that have encountered in the past $\left(N_{i}\right)$; increases the interactions strength with node $j$ by 1 (both the long term and the short term), and finally, the time of last contact is updated $\left(t_{i}=t\right)$.

We then define the aggregated interaction strength $s_{i j}$ between nodes $i$ and $j$ as $s_{i j}=\lambda_{i j}\left(\lambda_{i j}-\sigma_{i j}\right)$. Intuitively, the aggregated interaction strength is an indication of the frequency of long term interactions (proportionality to $\lambda_{i j}$ ), while penalizing spurious bursts of activity (proportionality to the difference between long and short time scales $\left(\lambda_{i j}-\sigma_{i j}\right)$ ).

Let define $u_{i j k}$ as node $i$ 's perceived utility of node $j$ to deliver a message to node $k$ as

$$
u_{i j k}=\frac{\lambda_{j k}\left(\lambda_{j k}-\sigma_{j k}\right)}{\lambda_{j k}\left(\lambda_{j k}-\sigma_{j k}\right)-\lambda_{i k}\left(\lambda_{i k}-\sigma_{i k}\right)},
$$

which represents utility of node $j$ to deliver a message to $k$ as seen by node $i$, normalized by the total utility. For values of $u_{i j k}>0.5$, we expect the node $j$ to do a better job than $i$ delivering the message to $k$. The utility $u_{i j k}$ is defined only when $\lambda_{i k}+\lambda_{j k}>0$, otherwise we set it as zero.

Analogously, let $u_{i j}$ be $i$ 's perceived utility of node $j$ to deliver a message to any node, defined as

$$
u_{i j}=\frac{\sum_{k \in N_{j}} \lambda_{j k}\left(\lambda_{j k}-\sigma_{j k}\right)}{\sum_{k \in N_{j}} \lambda_{j k}\left(\lambda_{j k}-\sigma_{j k}\right)+\sum_{k \in N_{i}} \lambda_{i k}\left(\lambda_{i k}-\sigma_{i k}\right)}
$$

Finally, node $i$ will forward to $j$ a message whose destination is $k$ iff

$$
\left\{\begin{aligned}
u_{i j k}>\frac{1}{2} & \wedge\left(\lambda_{i k}+\lambda_{j k}\right)>0 \\
u_{i j}>\frac{1}{2} & \wedge\left(\lambda_{i k}+\lambda_{j k}\right)=0
\end{aligned}\right.
$$


Notice that in order to calculate the utilities $u_{i j k}$, users only exchange their perceived interaction strength on node $k$, but never exchange the full contact list $N_{j}$. In order to obtain $N_{j}$, a node $i$ should probe $j$ for every possible value of $k$ in a short period of time (as the values $u_{i j k}$ decay with time). Then it is very easy for $j$ to identify such an attack and deny further communication with $i$.

\section{B. Assortative-Based Queue Control}

The heuristics in (6) do not completely achieve a balanced traffic distribution. The reason is that the routing decision is still a greedy maximization of the utility, with the forwarding biased towards the high connectivity nodes.

In order to counter this effect, we can again turn into sociology and observe the mechanisms by which people decide with whom they interact with. At the risk of being stereotypical, is it an empirically observed fact that the social status of someone's neighours is a good indicator of his status. The reason is that since social interactions require resources that are limited, humans carefully choose with whom they spend their resources with, and tend to allocate them so that the individual's utility is maximized. In other words, people of the same kind tend to interact together, and tend to disregard interactions from individuals from a lower social status.

For example, a big shot professor would allocate to time to review preliminary work from an equal peer, but it is unlikely to do the same for a graduate student. This behavior, known as assortativeness - or homophyly -, is one of the driving factors on the way individuals interact with each other [35], [36], [37], [38]. Assortativity is in fact what makes social networks different from other complex networks [39].

To capture the assortativeness in our algorithm, we define social status of a node $i$ in the DTN to be functionally equivalent to the size of the node's queue length $Q_{i}$. The queue length can be interpreted an indication that the node is often chosen to forward packets, and hence, is a measure of its popularity ${ }^{1}$. Since accepting to forward a message has a cost, nodes will only accept forward request from those nodes of equal or higher status. With assortative-based queue control, a node $i$ would forward a message directed $k$ through $j$ if any of the following conditions is met

$$
\left\{\begin{aligned}
\left(u_{i j k}>\frac{1}{2}\right) & \wedge\left(\lambda_{i k}+\lambda_{j k}\right)>0 \wedge\left(Q_{j} \leqslant Q_{i}\right) \\
\left(u_{i j k}=1\right) & \wedge\left(\lambda_{i k}+\lambda_{j k}\right)>0 \\
\left(u_{i j}>\frac{1}{2}\right) & \wedge\left(\lambda_{i k}+\lambda_{j k}\right)=0 \wedge\left(Q_{j} \leqslant Q_{i}\right)
\end{aligned}\right.
$$

High status nodes will be able to forward messages faster due to their privileged position, whereas low status nodes will have to find alternative paths. Since contact and social networks have a diversity of paths between two nodes [40], [41], constrains in the forwarding introduced by the assortativebased control queue does not necessarily implies a reduction of throughput (Section V). On the other hand, it does have a positive impact on the fairness of the routing algorithm (Section VI).

\footnotetext{
${ }^{1}$ Note that popularity does not necessarily means that the node is a high performer, but instead that the node is perceived to be very useful.
}

This mechanism of the queue size control to achieve load balance is analogous to the back pressure congestion control [30], which is applied to fields such as the Internet [32], [42], ATM and ethernet networks [43], and wireless sensor networks [31], among others.

\section{Privacy Considerations}

As social network-based routing algorithms become more sophisticated they require access to more information about users, and therefore, they raise rightful privacy concerns. For instance, Bubble and SimBet require access to the full list of contacts of a contact. While this is common practice in social networking sites, users of these systems explicitly aggregate their friends. This is not the case of DTNs, where contacts are aggregated upon proximity encounters. In addition, Bubble also requires access to the social group label the target user belongs to.

Having access to whom and how often a user has been interacting with is a security issue, and can be misused, for example, for profiling. While the authors' argument is to trade the privacy off in return for performance, we believe that it is preferable to obtain performance without giving sensitive information away.

\section{Contact Trace And Experimental Design}

\section{A. Contact Dataset}

Contract trace datasets [12], [13] are crucial to evaluate the performance of routing algorithms in DTNs. While large scale dataset (e.g. city-wide) are not available [44], the existing datasets allow the benchmarking of social network based routing algorithms. In this paper we use the MIT reality mining dataset [13], which is widely used in the literature, thus allowing a fair comparison of FairRoute with existing DTN routing algorithm. The MIT dataset was collected in the MIT campus, that represents a general purpose scenario better than other existing datasets such as Infocom 2006 [10].

In the MIT dataset users carried a cell phone with a software that logged contacts by proximity using the bluetooth discovery function. From the dataset we extracted the list of observed contacts: $\langle i, j, t\rangle$ where $i$ and $j$ are users, and $t$ is the time at which the interaction takes place. Some contacts might not last enough to allow data transfer between cell-phones. However, since we are only interested in patterns of humanto-human contact, we do not take this factor into consideration.

The MIT dataset provides contact trace information for 100 users over a period of over a year ${ }^{2}$. Figure 1(a) shows the time at which each contact takes place; there are three different stages, the first 652 interactions took place over the first 7 months, which might correspond to the development of the system. In the following 5 months the system showed 79840 interactions. In the last 4 months there was a decrease of the activity and only 33577 contacts were collected.

We partition the MIT dataset into 16 overlapping subsets each consisting in $35 \mathrm{~K}$ contacts and a relative offset of $5 \mathrm{~K}$ contacts. The utility of this partition is twofold. First, it allows

\footnotetext{
${ }^{2}$ The first interaction is logged on $01 / 01 / 2004$ and the last on $05 / 05 / 2005$.
} 


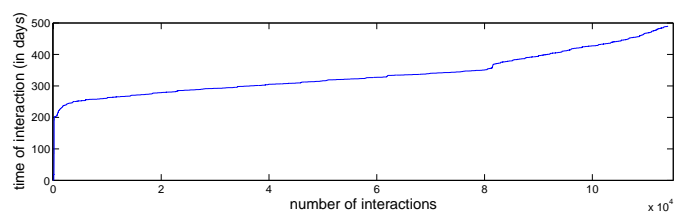

(a) Time (in days) at which the n-th contact takes place
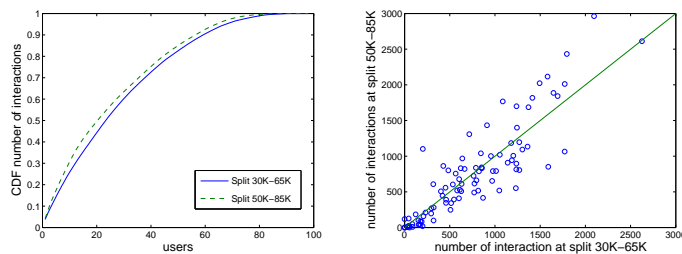

(b) Cumulative Distribution of (c) Scatter plot of the number contacts by user in two over- of contacts in two overlapping lapping subsets: $30 \mathrm{~K}$ to $65 \mathrm{~K}$ subsets. Each circle represents and $50 \mathrm{~K}$ to $85 \mathrm{~K}$ contacts. one of the 96 users that experience at least one contact

Fig. 1. Partition of the MIT reality mining project dataset in overlapping subsets

us to test the sensitivity of the algorithms against the existing variance of the contacts. Second, it minimizes the bias of the results toward the MIT dataset.

Figure 1(b) shows the comparison between the user's cumulative distribution in two partially overlapping subsets (from 30 to 65 thousand-th contact and from 50 to 85 thousandth contact). As expected, the aggregate contact distribution does not experience a lot of variance - as contacts take place between the same people in the same community. However, a look at the individual level (Fig. 1(c)) reveals that the number of contacts per user experiences a fair amount of variation. Note that both subsets have a $42 \%$ overlap.

\section{B. Reference Algorithms}

We evaluate the performance of FairRoute against wellknown algorithms in the literature.

- Epidemic routing [4] is a flooding multi-copy algorithm often used as a baseline for comparison because, while extremely inefficient in the number of forwards, it is optimal in terms of throughput. Furthermore, Epidemic routing is very adequate when exploring fairness, because its load distribution is caused solely by the network topology (i.e., it does not apply any heuristic to direct the forwarding).

- PROPHET [45] is widely used as the reference algorithm in terms of efficiency (e.g. in [16], [19]). PROPHET uses the history of contacts to calculate the probability of a node to deliver the message to the destination. Like FairRoute, it also takes the time between contacts into account to update its utility function. In our simulations, we use the parameters suggested by the author's of $\left\{P_{\text {init }}, \beta, \sigma\right\}=\{0.75,0.25,0.99\}$.

- SimBet [16] combines a decentralized version of betweenness centrality [46] and the probability of future collaboration [17]. To the best of our knowledge SimBet is the better algorithm to date efficiency-wise. For the simulations, and following the authors, we set the parameter $\alpha=0.5$.

The algorithm Bubble [19], which exploits the community structure found in social networks using a distributed clustering algorithm [47], was not included in our results because it requires the sender to know the address and the social group of the destination node, which is unfair to the other algorithms that only require the address. In addition, in the original paper, Bubble is not compared against the existing SimBet, but their comparison with PROPHET indicates that its performance is better than PROPHET but worse than SimBet.

\section{Experimental Design}

We run the four algorithms for each one of the 16 subsets of the MIT contact dataset. The first $5 \mathrm{~K}$ contacts of the subset are used to bootstrap the different algorithms. This warm up period is used to avoid introducing artifacts in the results due to the heuristics' different convergence time. After warm up phase, all the nodes send a message to every other node in the system, resulting in 9120 messages.

FairRoute's algorithm parameters are set to $r_{\lambda}=5 \times$ $10^{-4} h^{-1}$ and $r_{\sigma}=5 \times 10^{-3} h^{-1}$. Which correspond to the an average inter-contact time of 2000 and 200 hours respectively. These values will depend on the characteristic inter-contact time of the social network under scrutiny, for the MIT dataset they are extremely large because the contacts are very sparse (Fig. 1(a)). We also tested the parameter's sensitivity. Perturbations up to one order of magnitude did not show qualitative changes on the results of the algorithm.

\section{Throughrut AND EFFICIENCY Evaluation}

Maximizing the throughput, i.e., increasing the number of messages that reach their destination, is the goal of any routing algorithm. However, minimizing the number of forwards is also crucial to limit the resource consumption due to traffic: battery-life, storage, bandwidth. For this reason the efficiency, or the ratio between throughput and number of forwards, in combination with the throughput, are generally accepted as the metrics of choice for the routing algorithms.

Figure 2(a) shows the average throughput across the 16 subsets of the MIT dataset for all the algorithms. Table I shows the variance and numerical results summarized. The Epidemic routing yields the optimal throughput of $90 \%$ on average. SimBet and FairRoute deliver $81.1 \%$ and $79.9 \%$ of the messages respectively. PROPHET get a slightly worse performance with an average throughput of $74.5 \%$.

While all the algorithms achieve asymptotically good throughputs, it is important to note that epidemic is not only optimal in terms of throughput but also in delivery speed. As an example, if we consider the throughput after $10 \mathrm{~K}$ contacts, the throughput is $36.4 \%, 12.7 \%, 12.5 \%$ and $12.4 \%$ for Epidemic, SimBet, PROPHET and FairRoute respectively. As we can see, the three single-copy algorithms have a very similar performance, and the epidemic is clearly ahead of the rest.

However, as Figure 2(b) shows, FairRoute requires considerably less forwards. SimBet, PROPHET and epidemic require 1.37, 5.5 and 157 times more forwards than FairRoute. 


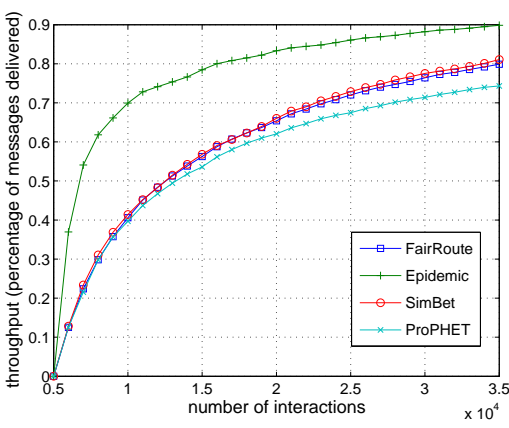

(a) Average throughput in percentage

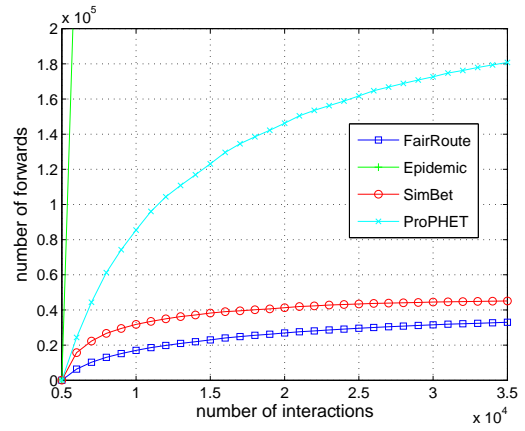

(b) Average number of forwards

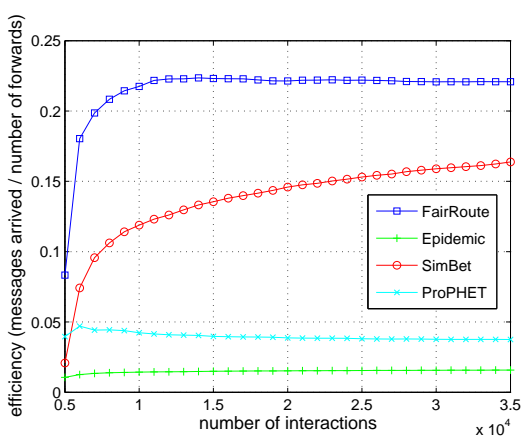

(c) Average efficiency (throughput / forwards)

Fig. 2. Throughput and Efficiency Evaluation

\begin{tabular}{|c|ll|ll|ll|}
\hline Algorithm & \multicolumn{2}{|l|}{ Throughput } & Forwards & \multicolumn{2}{l|}{ Efficiency } \\
& Avg $(\%)$ & StdDev & Avg & StdDev & Avg & StdDev \\
\hline Epidemic & 89.85 & 3.03 & 518790 & 18947 & 0.016 & 0.001 \\
PROPHET & 74.53 & 4.21 & 180790 & 26187 & 0.038 & 0.006 \\
SimBet & 81.08 & 4.74 & 45141 & 5389 & 0.166 & 0.022 \\
FairRoute & 79.90 & 3.94 & 32998 & 1492 & 0.221 & 0.007 \\
\hline
\end{tabular}

TABLE I

AVERAGE AND STANDARD DEVIATION FOR THROUGHPUT, FORWARDS AND EFFICIENCY AT THE END OF THE SIMULATION.

We would like to mention than SimBet [16] uses a different definition for forwards. They do not count as a forward the final hop by which the message arrives to destination (the handover). Also, they count as one forward when different messages can be bundled in the same data transfer session. This metric is somewhat misleading in terms of the cost associated to a message. Handovers messages still have a cost that needs to be accounted for when it send via wireless. The same happens to messages that are delivered in the same data transfer; the time of the transfer and its toll on the resources will be multiplied by the number of messages contained in the bundle. With SimBet's metric, FairRoute (with 11849 operations) still outperforms SimBet (with 13908 operations). Despite that, and for the sake of clarity, we take into account each individual message transfer as a forward.

Finally, Figure 2(c) displays the average efficiency. The most efficient algorithm is FairRoute followed by SimBet. PROPHET and epidemic routing fall behind due to their high costs in terms of number of forwards.

FairRoute's efficiency of 0.22 means that each message delivered to the destination requires, on average, 4.52 forward operations. Thus, assuming that the traffic generated by the routing algorithms is negligible when compared to the traffic generated by the forwards, one message of $100 \mathrm{~Kb}$ results in a $452 \mathrm{~Kb}$ of traffic to be handled by the participants of the DTN. In comparison, SimBet, PROPHET and Epidemic routing have a cost of $6.02,26.3$ and 62.5 times the cost of forwarding one message respectively.

\section{A. Variance Across Subsets}

As expected, the different subsets of the contact trace have a strong variance in throughput, number of forwards

\begin{tabular}{|c|l|l|l|l|}
\hline Algorithm & top-1 & top-10 & top-20 & Threshold \\
\hline Epidemic & 0.081 & 0.373 & 0.571 & 17 \\
PROPHET & 0.075 & 0.421 & 0.616 & 14 \\
SimBet & 0.134 & 0.539 & 0.706 & 9 \\
FairRoute & 0.046 & 0.275 & 0.452 & 24 \\
\hline
\end{tabular}

TABLE II

DISTRIBUTION OF FORWARDS BY USERS: TOP- $N$ CORRESPONDS TO THE FRACTION OF THE TOTAL NUMBER OF FORWARDS CARRIED OUT BY THE TOP $N$ USERS. THRESHOLD IS THE NUMBER OF USERS THAT CARRY OUT $50 \%$ OF THE ALL FORWARDS. THE TOTAL NUMBER OF USERS IS 96.

and efficiency (Table I). For instance, the throughput has a standard deviation of $3.03 \%$ for epidemic routing, $3.94 \%$ for FairRoute and $4.74 \%$ for SimBet. In the case of the number of forwards, the standard deviation accounts for differences of $11.9 \%, 4.5 \%$ and $3.7 \%$ for SimBet, FairRoute and epidemic routing respectively.

Such differences on the throughput and in the number of forwards clearly show the sensitivity of routing algorithms to the topology of the network of contacts. Despite the subsets belonging to the same social/contact network, and being clearly overlapped, the algorithms are sensitive to fluctuations in the contact patterns. We anticipate the need for large scale contact/social networks like [44] in order to explore the robustness of the routing algorithms for large-scale deployment.

\section{FAIRnESS Evaluation}

In the previous section we showed that FairRoute compares favorably to the state-of-the art DTN routing algorithms. Compared to SimBet, FairRoute obtains a 33\% increase in efficiently at a cost of just $1.2 \%$ loss in throughput. However, despite the good performance on the standard metric, the aim of FairRoute is neither throughput nor efficiency, but fairness.

Figure 3(a) shows the cumulative distribution of forwards for the four different algorithms. We can see that the natural load distribution due to the network topology - shown by the Epidemic routing - is already unbalanced: $50 \%$ of the traffic is handled by only 17 users, the top- 1 user deal single handedly with $8.1 \%$ of the traffic in the system and the top-10 users deals with $37 \%$.

While the perfectly fair load balance would be that $50 \%$ of traffic to be managed by $50 \%$ of users, it can only be 


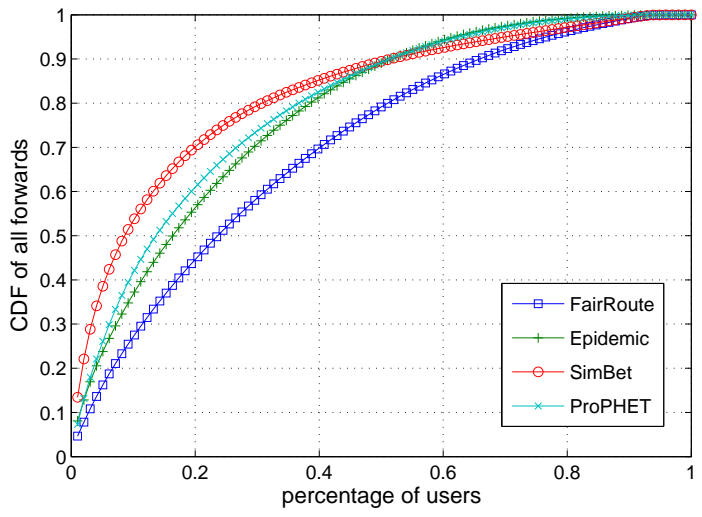

(a) Distribution of the total amount of forwards

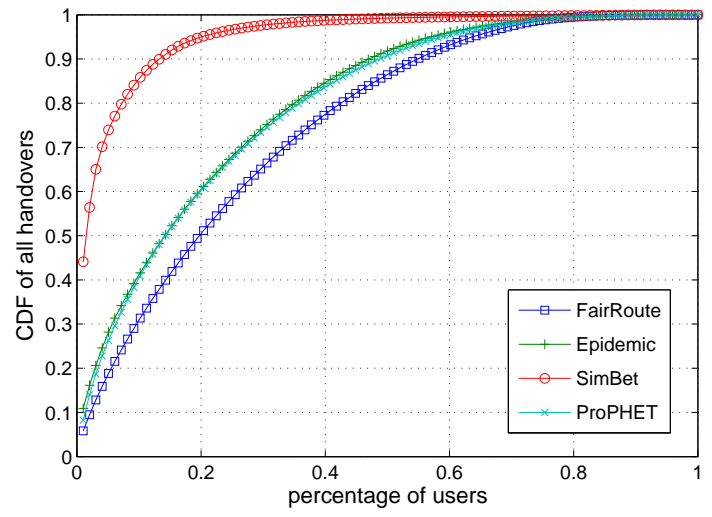

(b) Distribution of the total amount of handovers

Fig. 3. Fairness evaluation

achieved in a social network if there are no forwards but only handovers (i.e., each individual simply waits until it comes across the destination of the message - if ever). Since the perfect fairness is unrealistic, we set the baseline reference as the load distribution yield by the Epidemic routing, where the network topology is the only factor driving the load distribution.

As we discussed in Section II, the traffic load can only get worse for PROPHET and SimBET algorithms. In SimBet $50 \%$ of traffic goes through only 9 nodes, from which the top one handles $13.4 \%$. PROPHET does better with $50 \%$ of traffic being managed by 14 nodes and the top one node $7.5 \%$ of the traffic load. This behavior can be extrapolated to all routing algorithms based solely on best next hop heuristics as these heuristics contribute to the the already existing process of preferential attachment [25] of traffic due to the network topology. The fate of routing algorithms focused solely on best next hope heuristics is to increase performance (throughput and efficiency) at the expensed on unfair traffic distributions.

FairRoute, on the other hand, exhibits a load distribution that is more fair than the natural distribution of the epidemic routing. The assortativeness-based control queue is able to distribute the traffic more evenly among all the users, and therefore, leverage the congestion problems and failures discussed in section III. In FairRoute, 50\% of the traffic is handled by $25 \%$ of users, significantly better than the $9.3 \%$ of users for SimBet, the $14.5 \%$ of users for PROPHET and the $17.7 \%$ of the users for epidemic routing.

\section{A. Query Size Dynamics and Robustness}

Figure 4 displays the evolution of the queue size distribution over time for the SimBet, PROPHET and FairRoute algorithms. The white space between lines in the figure represents the fraction of messages in transit that has in its queue $\left(Q_{i}\right)$ at every interaction. During the initial stage of training (first $5 \mathrm{~K}$ contacts) there is no forwarding and each user's queue contains exactly 95 messages.

In the case of SimBet, (Figure 4(a)), it is revealing to see that right after the warmup period, two of the users accumulate most of the messages in transit in their queues. This behavior is inadvisable in terms of robustness, as a departure or a failure of one of those users would result in a loss of an important fraction of the messages waiting to be delivered.

The behavior of the queues of PROPHET (Fig. 4(b)) is more robust than SimBet's. The queue size distribution is quite homogeneously distributed among all users. The apparent discontinuity of the lines in the PROPHET is due to the high turn over of messages in the queue; PROPHET has one order of magnitude more forwards than SimBET and FairRoute. Consequently, messages exchange hands much more frequently and the queue distribution shows these fluctuations.

The FairRoute's queue size distribution (Fig. 4(c)) is more balanced than the other algorithms. This is due to its assortative-based queue control mechanism, which, as we saw in Fig. 3(a), also makes the traffic load more balanced.

With the traffic load distribution (Fig. 3(a)) and the queue size distribution (Fig. 4) we can explore the effect of fairness on robustness with a simple analysis. Let assume that an error happens during the forwarding process, and denote $e_{i}$ as the probability of node $i$ fails. Let $Q_{i, t}$ be the size of the queue of node $i$ at time $t$. We define $q_{i}$ as the average relative size of $i$ 's queue over time with respect to the total number of messages in the network, i.e., $q_{i}=\frac{\sum_{t} Q_{i, t}}{\sum_{t} \sum_{j} Q_{j, t}}$. Let us also denote the traffic load of node $i$ as $f_{i}$, which is the fraction of forwards carried out by $i$. Finally, let define the damage $D$ as $D=\sum_{i} e_{i} q_{i}$, i.e., the average cost in the fraction of messages in transit lost due a node's failure.

We consider three types of failures or errors:

- Random Error: the probability of failure is equally distributed between all nodes. Thus, $e_{i}=\frac{1}{96}$. Then, its trivial to see that the damage is $D=\frac{1}{96}$. A random error produces on average an loss of $1.04 \%$ of the messages regardless the routing algorithm.

- Load/cost error ${ }^{3}$ : in the case of an error, the probability of failure of a node $i$ is proportional to its traffic load

\footnotetext{
${ }^{3}$ The case of a node defecting because its perceived cost/benefit ratio is equivalent to the load error case.
} 


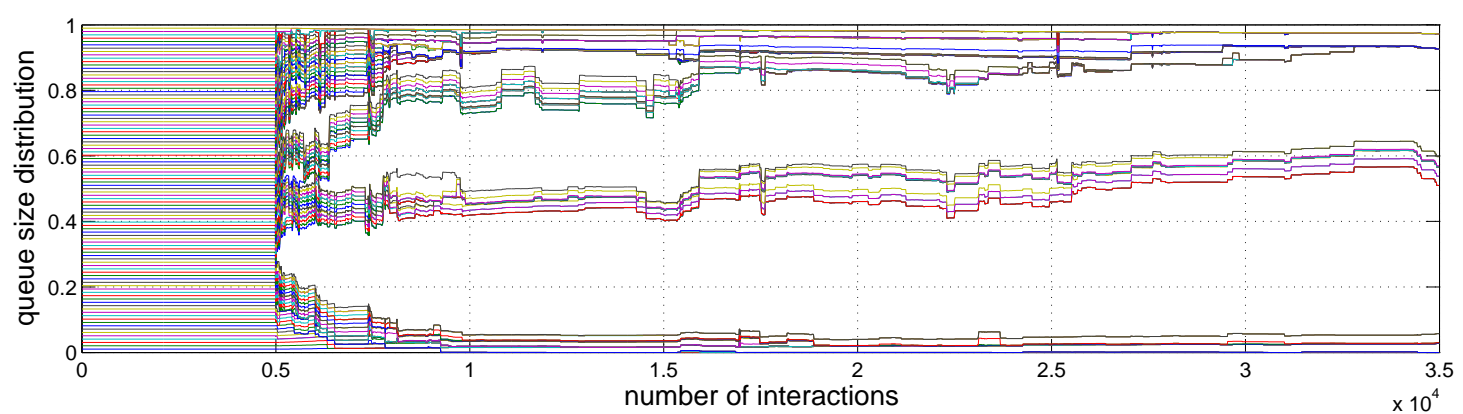

(a) SimBet algorithm

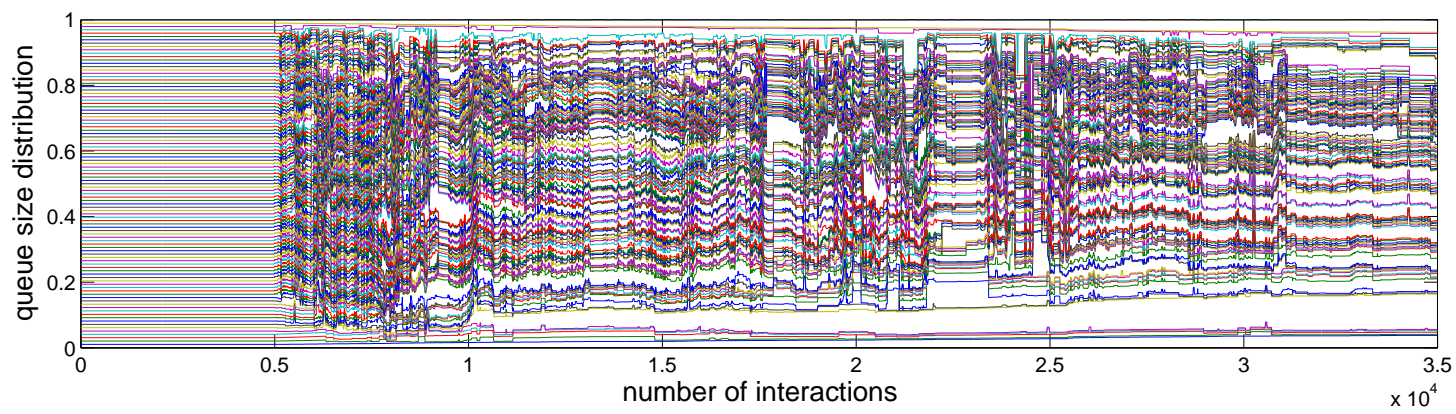

(b) PROPHET algorithm

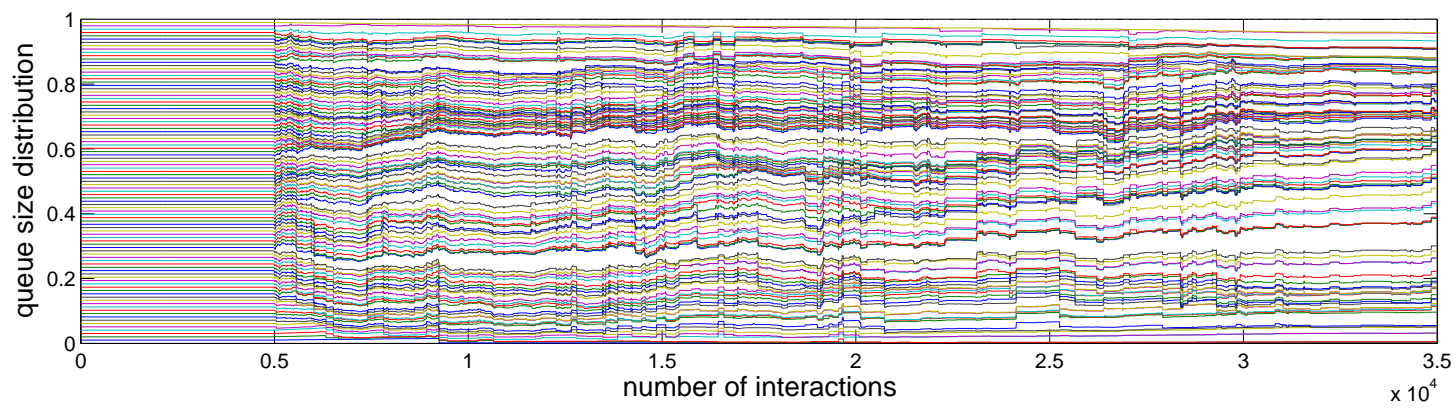

(c) FairRoute algorithm

Fig. 4. Evolution of the queue size distribution for all nodes (stacked). The proportion of messages in transit stored in user's $i$ queue corresponds to the white space between to lines. The proportion are stacked so that them sum one. The first $5 \mathrm{~K}$ contacts correspond to the warm up. In this period each user has $96^{-1}$ of the messages stored in the queue. After the $5 \mathrm{~K}$ contact, the forwarding starts and the queue size distribution evolves over time. This plot correspond to the $10 \mathrm{~K}-45 \mathrm{~K}$ split of the MIT contact dataset.

$f_{i}$ (resources are depleted proportionally to the traffic). Then, the average damage of one failure due to load is $D=\sum_{i} e_{i} q_{i}$, resulting in a damage of 5.81 for SimBet, 1.27 for PROPHET, and 2.21 for FairRoute. Also, note that the probability of individual errors increases with the traffic load, and so they will happen more often in algorithms that have more forwards. With PROPHET and SimBet performing 5.6 and 1.4 more forwards than FairRoute, the overall damage of load errors for FairRoute is considerably less than for the other algorithms.

- Attack: select the node that maximizes the damage $\left(\operatorname{argmax}\left(q_{i}\right)\right)$ and it will be 38.45\%, 5.46\% and 4.97\% for SimBet, PROPHET and FairRoute. Unbalanced load distributions are very fragile against attacks.

A more detailed analysis of robustness is outside the scope of this paper, at it has more complex ramifications in throughput and efficiency. Errors are not independent, and one failure can produce others in a cascade. However, the simple analysis performed above illustrates the fact that fairness is not optional, and hence, designers of DTN routing algorithms need to take fairness into consideration.

\section{B. Dynamic and Static Heuristics}

The subset of forwards that are also handovers have a special interest because they reveal another effect of the best next hop heuristics on the routing behavior. Figure 3(b) and Table III show who are the users that perform the last deliver of a message to the final destination. For the epidemic routing, the top- 5 users account for $28.8 \%$ of handovers. The same 5 users account for $26.80 \%$ of all handovers for PROPHET and $73.95 \%$ for SimBet.

These results illustrate one shortcoming of SimBet's heuris- 


\begin{tabular}{|c|l|l|l|l|}
\hline Algorithm & top-1 & top-5 & top-10 & Threshold \\
\hline Epidemic & 0.109 & 0.282 & 0.416 & 14 \\
PROPHET & 0.082 & 0.268 & 0.409 & 14 \\
SimBet & 0.441 & 0.739 & 0.858 & 2 \\
FairRoute & 0.058 & 0.188 & 0.313 & 20 \\
\hline
\end{tabular}

TABLE III

DISTRIBUTION OF HANDOVERS BY USERS: TOP- $N$ CORRESPONDS TO THE FRACTION OF THE TOTAL NUMBER OF HANDOVERS CARRIED OUT BY THE TOP $N$ USERS. THRESHOLD IS THE NUMBER OF USERS THAT CARRY OUT $50 \%$ OF THE ALL HANDOVERS. THE TOTAL NUMBER OF USERS IS 96.

\begin{tabular}{|c|ll|ll|ll|}
\hline Algorithm & \multicolumn{2}{|c|}{ Throughput } & \multicolumn{2}{c|}{ Forwards } & \multicolumn{2}{c|}{ Efficiency } \\
& Avg(\%) & Std & Avg & Std & Avg & Std \\
\hline Epidemic (A) & 89.75 & 3.07 & 499509 & 18250 & 0.016 & 0001 \\
PROPHET (A) & 67.47 & 4.62 & 78221 & 13575 & 0.077 & 0.014 \\
SimBet (A) & 67.35 & 4.62 & 28072 & 2180 & 0.219 & 0.018 \\
FairRoute & 79.90 & 3.94 & 32998 & 1492 & 0.221 & 0.007 \\
\hline
\end{tabular}

TABLE IV

AVERAGE AND STANDARD DEVIATION FOR THROUGHPUT, FORWARDS AND EFFICIENCY AT THE END OF THE SIMULATION. PROPHET, SIMBET AND EPIDEMIC INCORPORATE THE ASSORTATIVITY-BASED QUEUE PROPOSED BY FAIRROUTE

tic: the delivery utility does not age over time and therefore the heuristic does not forget. If a node's utility values are very stable against the changes of user's contact patterns a hill climbing strategy might be caught in a local mimina. Once there, it will not forward a message unless the actual destination is encountered. Thus, the most central nodes effectively become mailmen: the messages get to them very efficiently due to the high performance of SimBet's heuristics but once they reach the center of the network, the node will not deliver them to anyone but to the destination. Conversely, PROPHET and FairRoute's heuristics are dynamic and change according to the user's contacts fluctuation. The message will not be trapped in a local minima and therefore the handover distribution is similar to the handover distribution of the Epidemic Routing.

\section{EXPLORING FAIRROUTE'S ASSORTATIVENESS}

In the last section, we saw that the assortative-based queue control mechanism used by FairRoute yields a load distribution which is more balanced that the natural load distribution that arises from the structure of a complex network. It is reasonable to question whether this control mechanism can be seamlessly implemented in other algorithms to increase their fairness.

Figure 5(a) depicts the cumulative distribution of the forwards when all the algorithms use the same assortativeness principle as FairRoute, as described in Section III-B. We can see that assortativeness has no effect in the epidemic, as it follows a multi-copy policy. However, SimBet and PROPHET do show an increase on their fairness, which is now better than the natural load distribution. The efficiency of the routing algorithms is also increased: from 0.038 to 0.077 for PROPHET and from 0.166 to 0.219 for SimBet as shown in Table IV.

This increase in fairness and efficiency comes with a penalty in throughput, reduced from $81.08 \%$ to $67.35 \%$ for SimBet and from $74.33 \%$ to $67.47 \%$ for PROPHET. The loss of throughput is due to the inability of the SimBet's and PROPHET's heuristics to find alternative paths once the best next hop node stops accepting messages. FairRoute, on the other hand, is able to explore multiple paths between nodes [40], [41] more effectively and to keep its throughput of $73.95 \%$.

\section{CONCLUSIONS AND FUTURE WORK}

In this paper we presented FairRoute, an algorithm for routing in delay tolerant networks that achieves over $33 \%$ more efficiency than state-of-the art routing algorithms, without any loss in throughput. FairRoute achieves this performance by favoring peers that appear to be good candidates to deliver the message successfully to its target (perceived interaction strength), and by limiting communication with those nodes that have less importance in the network (assortativity).

The most important contribution of FairRoute, however, is that unlike other routing algorithms inspired in social mechanisms, our algorithm distributes the traffic load more evenly. While in SimBet the top 10 users in the MIT reality mining project dataset manage $54 \%$ of the traffic, they account for only $27.5 \%$ of the traffic when FairRoute is used. Our algorithm achieves a load distribution that is even more balanced than the natural load distribution that arises from the social network-like structure of the MIT dataset, in which the top 10 users manage the $37 \%$ of the traffic.

At this point we would like to propose a last thought experiment. Let assume that the reader is one of the top users in the MIT dataset, i.e., the reader plays a central role in the community and therefore a lot of traffic goes through his/her cell-phone. Let also assume that the messages size is $100 \mathrm{~Kb}$ (e.g. an MMS, or an email with an attachment). Then, on average, every single message sent between any two individuals in the network would generate a total traffic of $452 \mathrm{~Kb}, 602 \mathrm{~Kb}$ and $6230 \mathrm{~Kb}$ for FairRoute, SimBet and Epidemic routing respectively. The cost for the reader, a central node is the social network, is of $20 \mathrm{~Kb}$ with FairRoute, $80 \mathrm{~Kb}$ with SimBet, and as much as $500 \mathrm{~Kb}$ with Epidemic routing for every single message sent in the network.

Needless to say that Epidemic routing is prohibitively expensive. The question remaining is whether SimBet, or even FairRoute, are prohibitive as well. Although FairRoute reduces the cost of SimBet by a factor of four, it is still carries a significant cost for the central users of the network that they might not be willing to assume. This reflexion does not intent to downplay the Delay Tolerant Networks, an area if immense potential, but to highlight the fact that there is a need to switch the metrics of choice from raw efficiency to fairness and load balancing in order to obtain more realistic routing algorithms.

\section{ACKNOWLEDGEMENTS}

We thank Elizabeth Daly and Heiko Yoneki for kindly answering our questions regarding SimBet and BubbleRAP.

\section{REFERENCES}

[1] S. Keshav. Why cell phones will dominate the future internet. SIGCOMM Comput. Commun. Rev., 35(2):83-86, 2005.

[2] K. Fall. A delay-tolerant network architecture for challenged internets. In Proc. SIGCOMM, 2003.

[3] M. Grossglauser and D. Tse. Mobility increases the capacity of ad hoc wireless networks. IEEE/ACM Transactions on Networks, 10(4):477486, 2002. 


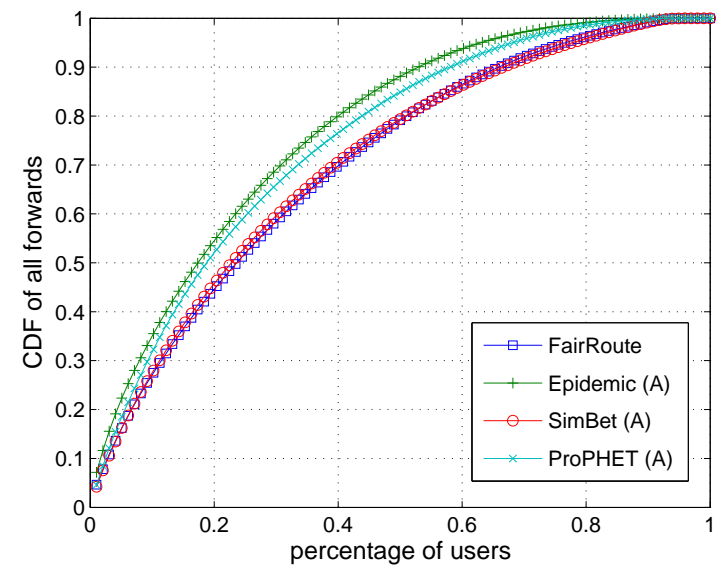

(a) Distribution of the total amount of forwards

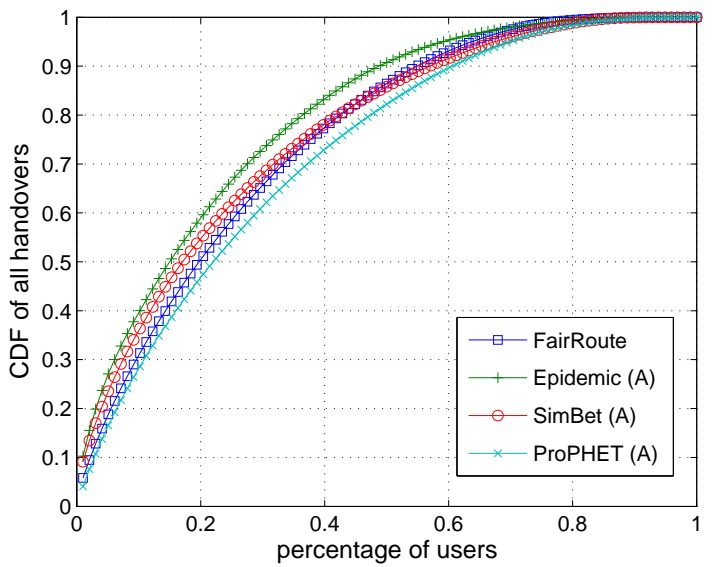

(b) Distribution of the total amount of handovers

Fig. 5. Fairness evaluation. The routing algorithms are modified version of SimBet, PROPHET and Epidemic routing with the FairRoute's assortativity-based queue control.

[4] A. Vahdat and D. Becker. Epidemic routing for partially connected ad hoc networks. Technical Report CS-200006, Duke University, 2000.

[5] Q. Li and D. Rus. Sending messages to mobile users in disconnected ad hoc networks. In Proc. of MobiCom, pages 44-55, 2000.

[6] S. Jain, K. Fall, and R. Patra. Routing in a delay tolerant network. SIGCOMM Comput. Commun. Rev., 34(4):145-158, 2004.

[7] J. Burgess, B. Gallagher, B. Jensen, and B.N. Levine. Routing for vehicle-based disruption-tolerant networking. In Proc. of Infocom, 4:1688-1698, 2006

[8] M. Grossglauser and M. Vetterly. Locating nodes with ease: last encounter routing in ad hoc networks through mobility difussion. In Proc. of Infocom, 3(4):1954-1964, 2003.

[9] T. Karagiannis, J.-Y.L. Boudec, and M. Vojnovic. Power-law and exponential decay of intercontact time between mobile devices. Proc. of ACM MobiCom, 2007.

[10] A. Chaintreau, P. Hui, J. Crowford, C. Diot, J. Scott, and R. Gass. Impact of human mobility on oportunistic forwarding algorithms. IEEE Trans. Mob. Comp., 6(6):606-620, 2007.

[11] P. Costa, C. Mascolo, M. Musolesi, and G.-P. Picco. Socially-aware routing for publish-subscribe in delay-tolerant mobile ad hoc networks. IEEE Journal on Selected Areas in Communications, 26(5), 2008.

[12] Haggle Project. http://www.haggleproject.org/.

[13] N. Eagle and A. Pentland. Reality mining: sensing complex social systems. Personal and Ubiquitous Computing, 10(4):255-268, 2006.

[14] E. Yoneki, P. Hui, and J. Crowcroft. Distinct types of hubs in human dynamic networks. EuroSys Workshop on Social Network Systems, 2008.

[15] A. Chaintreau, P. Fraigniaud, and E. Lebhar. Opportunistic spatial gossip over mobile social networks. 6(6):606-620, 2007.

[16] E. Daly and M. Haahr. Social network analysis for routing in disconnected delay-tolerant manets. In Proc. of ACM MobiHoc, 2007.

[17] D. Liben-Nowell and J. Kleinberg. The link prediction problem for social networks. In Proc. of CIKM, 2003.

[18] L.C. Freeman. A set of measuring centrality based on betwenness. Sociometry, 40:35-41, 1977.

[19] P. Hui, J. Crowcroft, and E. Yoneki. Bubble rap: Social based forwarding in delay tolerant networks. In Proc. of MobiHoc, 2008.

[20] M.E.J. Newman and M. Girvan. Finding and evaluating community structure. Phys. Rev. E, 69:026113, 2004.

[21] N. Sastry. Folksonomy-based reasoning in opportunistic networks. In Proc. of CoNEXT, page 49, 2007.

[22] S. Guo and S. Keshav. Fair and efficient scheduling in data ferrying networks. In Proc. of ACM/CoNEXT, 2007.

[23] A. Balasubramanian, B.N. Levine, and A. Venkataramani. Dtn routing as a resource allocation problem. In Proc. of SIGCOMM, 2007.

[24] A. Mtibaa, A. Chaintreau, J. LeBrun, E. Oliver, A.-K. Pietilainen, and C. Dio. Are you moved by your social network application? In Proc. of SIGCOMM Workshop on Social Networks, 2008.

[25] R. Albert and A.-L. Barabási. Statistical mechanics of complex networks. Review of Modern Physics, 74:47-97, 2002.
[26] M.E.J. Newman. The structure and function of complex networks. SIAM Review, 45(2):167-256, 2003.

[27] P. Holem and B.J. Kim. Vertex overload breakdown in evolving networks. Phys. Rev. E, 65:066109, 2002.

[28] R. Albert, H. Jeong, and A.-L. Barabási. The internet's achilles' heel Error and attack tolerance in complex networks. Nature, 406:378-381, 2000.

[29] E. Fehr and S. Gachter. Fairness and retaliation: The economics of reciprocity. Journal of Economic Perspecives, 14(3):159-182, 2000.

[30] L. Tassiulas. Adaptive back-pressure congestion control based on local information. IEEE Transaction on Automatic Control, 40(2):236, 1995.

[31] B. Hull, K. Jamieson, and H. Balakrishnan. Mitigating congestion in wireless sensor networks. In Proc. of 2nd International Conference on Embedded Networks Sensor Systems, pages 134-147, 1994.

[32] S. Floyd. Tcp and explicit congestion notification. ACM Computer Communication Review, 24(5):10-23, 1994.

[33] D.J. Watts and P. Sheridan-Dodds. Influentials, networks, and public opinion formation. Journal of Consumer Research, 34(4):441-458, 2007.

[34] M. Granovetter. The strength of weak ties. American Journal of Sociology, 78, 1973.

[35] P. Blau. Exchange and Power in Social Life. Wiley, New York, 1964.

[36] R.V. Gould. Collective action and network structure. American Sociological Review, 58:182-196, 1993.

[37] N. Mark. Beyond individual differences: Social differentiation from first principles. American Sociological Review, 63:309-330, 1998.

[38] N. Lin. Social Capital: A theory of social structure and action. Cambridge University Press, 2001.

[39] M.E.J. Newman and J. Park. Why social networks are different from other types of networks. Phys. Rev. E, 68:036122, 2003.

[40] V. Erramilli, A. Chaintreau, M. Crovell, and C. Diot. Diversity of forwarding paths in pocket switched networks. In Proc. of IMC, 2007.

[41] D.J Watts. Network dynamics and the small world phenomenon. Americal Journal of Sociology, 105(2):493-527, 1999.

[42] Sally Floyd and Van Jacobson. Random early detection gateways for congestion avoidance. IEEE/ACM Transactions on Networking, 1:397413, 1993.

[43] IEEE. IEEE, ed., 802.3 standard. IEEE, 2002, ch. 2, annex 31b: MAC control PAUSE operation

[44] M.C. González, C.A. Hidalgo, and A.-L. Barabási. Understanding individual human mobility patterns. Nature, 453:779-782, 2008.

[45] A. Lindgren, A. Doria, and O. Schelen. Probabilistic Routing in intermittently connected networks, volume 3126, pages 239-254. 2004.

[46] P.V. Marsden. Egocentric networks and sociometric measures of network centrality. Social Networks, 24:407-422, 2002.

[47] P. Hui and E. Yoneki. Distributed community detection in delay tolerant networks. In Proc. of MobiHoc, 2007. 